$12-2004$

\title{
Fast Measurements of Flow Through Mitral Regurgitant Orifices With Magnetic Resonance Phase Velocity Mapping
}

\author{
Haosen Zhang \\ Cleveland State University \\ Sandra S. Halliburton \\ Cleveland State University \\ Richard D. White \\ Cleveland Clinic Foundation
}

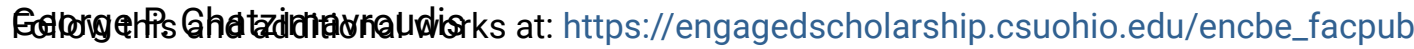

I'veland State University

Part of the Biomedical Engineering and Bioengineering Commons, and the Transport Phenomena

Commons

How does access to this work benefit you? Let us know!

Publisher's Statement

The final publication is available at Springer via http://dx.doi.org/10.1007/s10439-004-7815-4

\section{Original Citation}

Zhang, H., Halliburton, S. S., White, R. D., , \& Chatzimavroudis, G. P. (2004). Fast Measurements of Flow through Mitral Regurgitant Orifices with Magnetic Resonance Phase Velocity Mapping. Annals of Biomedical Engineering, 32(12), 1618 - 1627. doi:10.1007/s10439-004-7815-4

\section{Repository Citation}

Zhang, Haosen; Halliburton, Sandra S.; White, Richard D.; and Chatzimavroudis, George P., "Fast Measurements of Flow Through Mitral Regurgitant Orifices With Magnetic Resonance Phase Velocity Mapping" (2004). Chemical \& Biomedical Engineering Faculty Publications. 78.

https://engagedscholarship.csuohio.edu/encbe_facpub/78

This Article is brought to you for free and open access by the Chemical \& Biomedical Engineering Department at EngagedScholarship@CSU. It has been accepted for inclusion in Chemical \& Biomedical Engineering Faculty Publications by an authorized administrator of EngagedScholarship@CSU. For more information, please contact library.es@csuohio.edu. 


\title{
Fast Measurements of Flow through Mitral Regurgitant Orifices with Magnetic Resonance Phase Velocity Mapping
}

\author{
HaOSEn Zhang,,${ }^{1,2,3}$ SANDra S. HAlliburton, ${ }^{1,2}$ Richard D. White, ${ }^{2}$ and GeORGe P. Chatzimavroudis ${ }^{1,2}$ \\ ${ }^{1}$ Laboratory of Biofluid Mechanics and Cardiovascular Imaging, Department of Chemical and Biomedical Engineering, Cleveland State \\ University, Cleveland, $\mathrm{OH} ;{ }^{2}$ Section of Cardiovascular Imaging, Division of Radiology, The Cleveland Clinic Foundation, Cleveland, \\ $\mathrm{OH}$; and ${ }^{3}$ Cardiovascular Imaging Laboratory, Mallinckrodt Institute of Radiology, Washington University School of Medicine,
}

St. Louis, MO

\begin{abstract}
Magnetic-resonance (MR) phase velocity mapping $(\mathrm{PVM})$ shows promise in measuring the mitral regurgitant volume. However, in its conventional nonsegmented form, MR-PVM is slow and impractical for clinical use. The aim of this study was to evaluate the accuracy of rapid, segmented $k$-space MR-PVM in quantifying the mitral regurgitant flow through a control volume (CV) method. Two segmented MR-PVM schemes, one with seven (seg-7) and one with nine (seg-9) lines per segment, were evaluated in acrylic regurgitant mitral valve models under steady and pulsatile flow. A nonsegmented (nonseg) MR-PVM acquisition was also performed for reference. The segmented acquisitions were considerably faster $(<10 \mathrm{~min})$ than the nonsegmented ( $>45 \mathrm{~min}$ ). The regurgitant flow rates and volumes measured with segmented MR-PVM agreed closely with those measured with nonsegmented MR-PVM (differences $<5 \%, p>0.05$ ), when the $\mathrm{CV}$ was large enough to exclude the region of flow acceleration and aliasing from its boundaries. The regurgitant orifice shape (circular vs. slit-like) and the presence of aortic outflow did not significantly affect the accuracy of the results under both steady and pulsatile flow $(p>0.05)$. This study shows that segmented $k$-space MR-PVM can accurately quantify the flow through regurgitant orifices using the $\mathrm{CV}$ method and demonstrates great clinical potential.
\end{abstract}

Keywords-MRI, Segmented $k$-space, Mitral regurgitation, Velocity measurements, Flow quantification, Control volume.

\section{INTRODUCTION}

Magnetic-resonance (MR) phase velocity mapping (PVM) is widely used clinically to provide blood flow information. The technique is based on the principle that by using the proper bipolar magnetic field gradients in the direction of interest, the velocity of hydrogen nuclei in that direction can be encoded into the phase of the detected signal. ${ }^{19}$ MR-PVM has been extensively evaluated as a flow quantification technique in vitro and clinically, showing high ac- curacy and precision. ${ }^{1,2,4,6,7,9,12,13,14,17,18,20,26}$ It is the only clinical technique capable of providing three-directional velocity information at any point in the flow field in an imaging slice taken in any orientation.

Regurgitation is a common and serious type of heart valve disease. It degrades cardiac function and efficiency and leads to cardiac failure and death in severe cases if untreated. Diagnosis of the disease has been mainly qualitative. ${ }^{8,21,24,27}$ Initial quantitative approaches were indirect by estimating the regurgitant amount from volumetric measurements of the ventricles during the cardiac cycle ${ }^{23}$ however, this method is not valid in concomitant valve insufficiency. The need for reliable quantitative assessment of regurgitation has led to the development of approaches that can directly measure the regurgitant flow. Doppler ultrasound techniques, such as the proximal iso-velocity surface area (PISA) method, ${ }^{22}$ were developed and evaluated. Despite its potential, PISA can be affected by geometric uncertainties and the complication of the flow environment in the moving left ventricle during systole., ${ }^{3,11}$ One of the limitations of Doppler ultrasound is that it provides only one component of the blood velocity vector (along the direction of the ultrasonic beam). In contrast, MR-PVM can measure all three spatial components of the velocity vector. This led to the development of a multislice control volume (CV) MRPVM method (Fig. 1) for the quantification of the regurgitant flow. ${ }^{5,28}$ The CV method involves the acquisition of a number of contiguous imaging slices in the valve region. All three components of the velocity vector are acquired per slice. By considering a CV encompassing the regurgitant orifice in the ventricular side and calculating the net inflow through the surfaces of the $\mathrm{CV}$, the regurgitant flow can be calculated via the mass conservation principle. This approach was evaluated using conventional nonsegmented MR-PVM. ${ }^{5}$ Despite the accuracy found, the main problem of this approach is that it requires $1 \mathrm{~h}$ or even longer for a complete measurement of the regurgitant volume, making it clinically impractical. However, with the development 

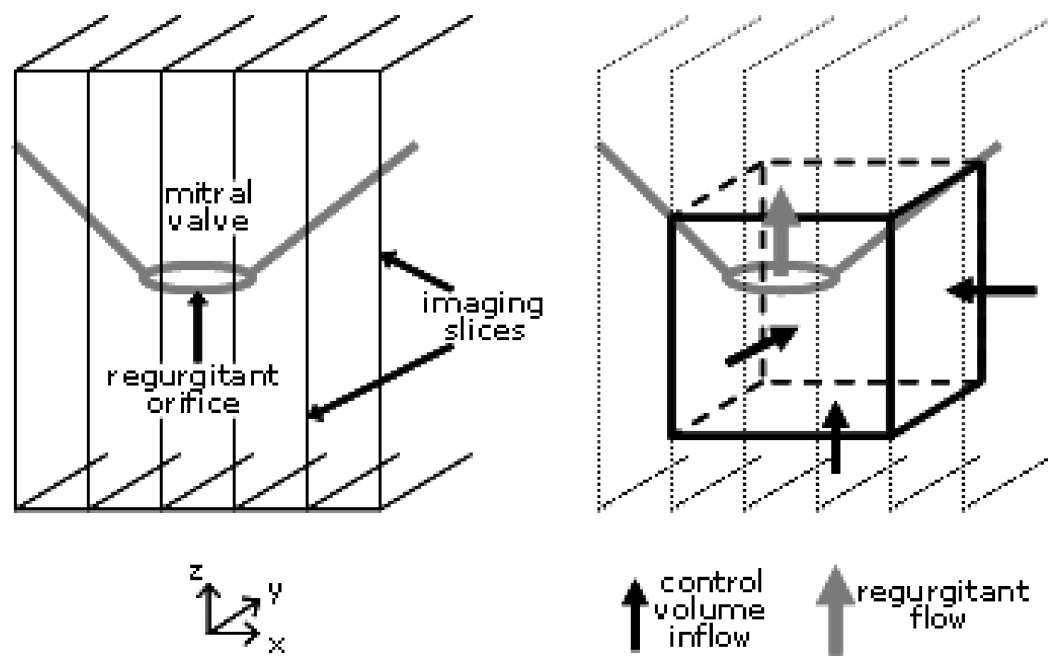

FIGURE 1. The CV method: a number of contiguous imaging slices are placed in the mitral valve region with the central slices passing through the center of the regurgitant orifice (a). All three spatial components of the velocity vector in each slice are acquired and a CV is considered to encompass the orifice (b). Based on the mass conservation principle, the net amount of fluid that enters the five faces of the $\mathrm{CV}$ from the ventricular side will be equal to the amount of fluid that flows through the regurgitant orifice.

of segmented $k$-space MR-PVM techniques, ${ }^{10,15,16,25,30}$ the $\mathrm{CV}$ method may become the clinical method of choice for a reliable measurement of the amount of blood that regurgitates through the leaky mitral valve.

MR-PVM is applied using a gradient-echo sequence with a bipolar velocity-encoding gradient in the desired direction for velocity measurement. Using the electrocardiogram (ECG), the MR scanner is triggered to acquire multiple images corresponding to multiple time-phases throughout the cardiac cycle. For each time-phase, the corresponding image is reconstructed from the signal acquired during many sequential cardiac cycles. For an image of $256 \times 256$ pixels, the signal should normally be acquired 256 times. Each time the signal is acquired, the sampled data is used to fill one line of the frequency domain ( $k$-space).

Using conventional nonsegmented MR-PVM, only one of these $k$-space lines is acquired per time-phase during a single cardiac cycle. Therefore, it takes 256 cardiac cycles to acquire all lines necessary to reconstruct the image. However, $k$-space segmentation can dramatically reduce the data acquisition time. Instead of acquiring only one $k$-space line for each time-phase of the cardiac cycle and then waiting until the next heart beat to acquire the next $k$-space line, a segment of several $k$-space lines can be acquired very rapidly per time-phase during each heart beat. The required number of $k$-space lines is filled in fewer cardiac cycles, thereby reducing the acquisition time. The larger the number of lines assigned per segment, the faster the acquisition, but the lower the temporal resolution of the acquired data.

The reduction in scan time makes the CV MR-PVM method promising for clinical implementation. The accuracy of segmented $k$-space MR-PVM for through-plane and in-plane velocity measurements in a slice was recently evaluated in vitro ${ }^{30,31}$ and clinically, ${ }^{6}$ showing high levels of reliability. Therefore, the aim of this study was to evaluate the accuracy of the CV MR-PVM method, with $k$ space segmentation of the data acquisition, in quantifying the regurgitant flow through orifices that simulated mitral regurgitant orifices, under a variety of geometric and flow conditions.

\section{METHODS}

Steady and pulsatile water flow experiments were performed in a $1.5 \mathrm{~T}$ Siemens Sonata whole-body MR scanner (Siemens Medical Solutions, Erlangen, Germany) with a maximum gradient strength of $40 \mathrm{mT} / \mathrm{m}$. A cylindrical acrylic left ventricular model (Fig. 2) was used for the experiments. The model had an opening to allow the insertion of cone-shaped mitral valve models. It also included an aor-

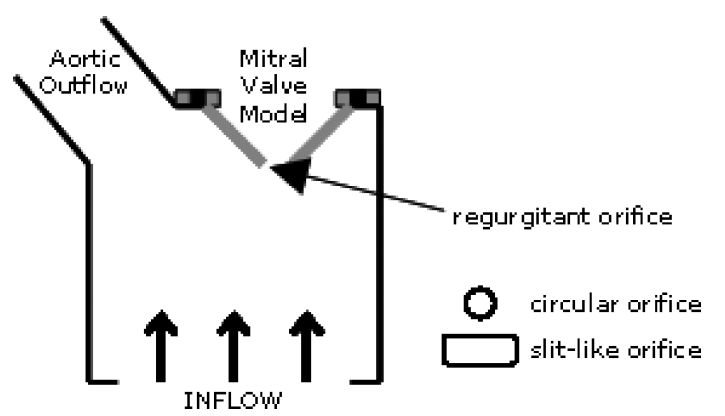

FIGURE 2. Left ventricular model with the regurgitant mitral valve model and the aortic outflow. Flow entered the model from its "apical" side. 
TABLE 1. Experimental conditions for mitral regurgitation study under steady flow.

\begin{tabular}{lccr}
\hline Orifice type & Circular & Circular & \multicolumn{1}{c}{ Slit-like } \\
\hline Orifice size & $3 \mathrm{~mm}(\mathrm{ID})$ & $\begin{array}{c}5 \mathrm{~mm}(\mathrm{ID}) \\
90^{\circ}\end{array}$ & $4 \times 10 \mathrm{~mm}^{2}$ \\
Cone-apex angle & & & \\
Regurgitant flow rate & $1.0(2.4)$ & $2.5(2.1)$ & $4.5(2.0)$ \\
$\quad(\mathrm{l} / \mathrm{min})$ & $1.5(3.5)$ & $4.0(3.4)$ & $7.0(3.2)$ \\
In parenthesis: & $2.0(4.7)$ & $5.5(4.7)$ & $11.0(5.0)$ \\
$\quad$ Orifice velocity (m/s) & & & \\
Aortic outflow $(\mathrm{l} / \mathrm{min})$ & & $0-25$ & \\
\hline
\end{tabular}

tic outflow tract to better simulate the physiologic systolic ventricular flow environment. Three regurgitant valve models were used: two with circular orifices [ 3 and $5 \mathrm{~mm}$ of inner diameter (ID)]; and one with a slit-like orifice $4 \times 10 \mathrm{~mm}^{2}$ (Fig. 2). A range of steady flow rates and pulsatile flow volumes was investigated to simulate a range of regurgitant flow conditions. Pulsatile flow was generated by a computer-controlled piston pump (SuperPump, SPS 3891 Vivitro Systems Inc., Victoria, British Columbia, Canada). Flow entered the ventricular model from its "apical" side and exited through the aorta and the regurgitant valve model. The shape and size of the regurgitant orifice and the corresponding flow conditions are listed in Tables 1 and 2 for steady and pulsatile flow, respectively. Under steady flow conditions, the true flow rates were known via precalibrated rotameters (Dakota Instruments Inc., Orangeburg, $\mathrm{NY}$ ), whereas under pulsatile flow conditions, the flow profiles were known with precalibrated, MR-compatible, brass transit-time ultrasonic flow probes (Transonic Systems Inc., Ithaca, NY).

The ventricular model was placed in a saline-filled container to assure adequate signal detection for tuning and was inserted into the bore of the scanner so that the mitral regurgitant orifice was at the iso-center of the scanner. A phased-array receiver coil was used to cover the test section to improve image quality. Initial true-FISP scout images in all three spatial orientations (axial, coronal, and sagit-

TABLE 2. Experimental conditions for mitral regurgitation study under pulsatile flow.

\begin{tabular}{lccc}
\hline Orifice type & Circular & Circular & Slit-like \\
\hline Orifice size & $3 \mathrm{~mm}$ (ID) & $5 \mathrm{~mm}$ (ID) & $4 \times 10 \mathrm{~mm}^{2}$ \\
Cone-apex angle & & $90^{\circ}$ & \\
Regurgitant volume & 10 & 30 & 40 and 60 \\
$\quad(\mathrm{ml}$ per cycle) & & & \\
$\begin{array}{l}\text { Estimated range of peak } \\
\quad \text { regurgitant orifice }\end{array}$ & & $2.5-7.0$ & \\
$\quad$ velocity (m/s) & $2.5-7.0$ & \\
Aortic outflow volume & & & \\
$\quad(\mathrm{ml}$ per cycle) & & 40 or 60 & \\
Rate (cycles/min) & \multicolumn{2}{c}{60} \\
\hline
\end{tabular}

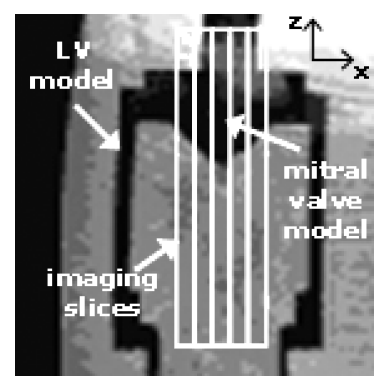

FIGURE 3. Coronal scout image showing the acrylic model of the left ventricle with the cone-shaped mitral valve model and the placement of the stack of the five contiguous slices for the three-directional velocity measurements.

tal) were acquired to determine the position of the mitral regurgitant orifice (Fig. 3). After the scout images, threedirectional velocity acquisitions were performed using a nonsegmented gradient-echo sequence (nonseg) and two segmented sequences with seven ( $\operatorname{seg}-7)$ and nine ( $\operatorname{seg}-9)$ $k$-space lines per segment. The selection of these segmented sequences was the result of our recent evaluation of these segmentation schemes in straight tubes. ${ }^{30}$ The nonseg acquisitions were performed to provide the reference data in addition to flowmeter readings. For each sequence, five contiguous sagittal imaging slices (Fig. 3) were acquired (flip angle of $30^{\circ}$, slice thickness of $5 \mathrm{~mm}$ ) with the central slice placed at the center of the regurgitant orifice. A $75 \%$ rectangular $250 \mathrm{~mm}$ field of view (FOV) was used for the nonsegmented acquisitions and a $56 \%$ rectangular $250 \mathrm{~mm}$ FOV was used for the segmented acquisitions. After interpolation, the voxel size was $1.0 \times 1.0 \times 5.0 \mathrm{~mm}^{3}$ in all cases. The velocity-encoding value was $80-250 \mathrm{~cm} / \mathrm{s}$, depending on the magnitude of the flow. The echo time (TE) was varied between 2.3 and $4.7 \mathrm{~ms}$ (shortest possible based on other imaging parameters). In pulsatile flow, the acquisitions were gated using a triggering signal synchronized with the flow waveform signal driving the piston pump. Gating resulted in $33,15-16$, and 11-12 time-phases per cycle for the nonseg, seg-7, and seg-9 sequences, respectively. The duration of each nonseg scan (one slice, one velocity component) was approximately $3 \mathrm{~min}$, whereas the durations of the seg-7 and $\operatorname{seg}-9$ scans were 20 and $16 \mathrm{~s}$, respectively.

Each MR-PVM acquisition produced magnitude and phase images (Fig. 4), which were transferred to a workstation (Ultra-10, SUN Microsystems Inc., Palo Alto, CA). The images were visualized using Transform (Version 3.4, Research Systems Inc., Boulder, CO). A computer program was developed to convert the phase values of the phase images to velocity values based on the linearity between signal phase and proton velocity. Then, the program selected a range of $\mathrm{CV}$ sizes encompassing the regurgitant orifice in the ventricular model. For each selected CV size, the program calculated the flow rate of the fluid that passed through the five surfaces of the $\mathrm{CV}$ in the ventricular side, 


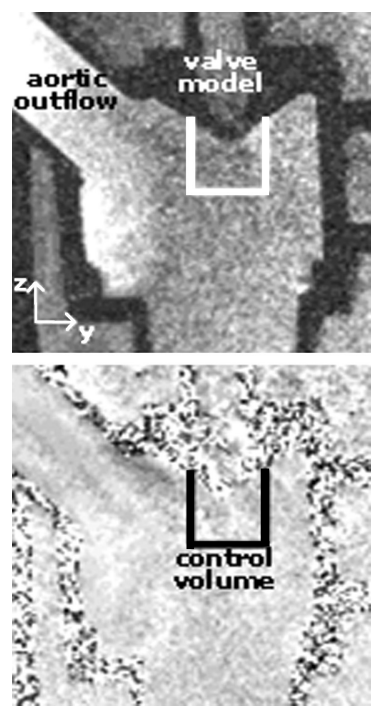

FIGURE 4. Magnitude (top) and phase (bottom) images acquired from the central slice of the slice stack. An example of a CV selection is shown (2-D demonstration).

by summing the products of "pixel velocity" and "pixel area". Based on the principle of mass conservation, this flow rate should be equal to the flow rate crossing the sixth face of the CV (located in the atrial side of the model), which is the regurgitant flow rate (Fig. 1). In pulsatile flow, integration of the flow rate curve over systole provided the regurgitant flow volume. In this text, the three dimensions of the $\mathrm{CV}$ are defined as (refer to Figs. 1, 3, and 4): $x, \mathrm{CV}$ size in the left-right direction; $y, \mathrm{CV}$ size in the anterior-posterior direction; and $z, \mathrm{CV}$ size in the foot-head direction.

Statistical analysis (paired $t$-tests, analysis of variance, sign tests, and Kruskal-Wallis tests) was performed to compare the steady and pulsatile flow results with the true values for all sequences, and to determine the effect of sequence type, aortic outflow, and orifice geometry, using Minitab (Version 13, Minitab Inc., State College, PA). A p-value $<0.05$ indicated significant difference.

\section{RESULTS}

Figure 4 shows a pair of magnitude and phase (velocity) images of the acrylic ventricular model in the sagittal direction. The ventricular cavity, the aortic outflow tract, and the cone-shaped regurgitant mitral valve model can be clearly identified. The images were of good visual quality that ensured reliability in identifying the valve and, thus in quantitatively analyzing the data.

Representative steady flow results can be seen in Fig. 5, which shows the calculated regurgitant flow rates through the $5 \mathrm{~mm}$ ID circular regurgitant orifice using the $\mathrm{CV}$ method for a range of $z$ (foot-head) and $y$ (anterior-
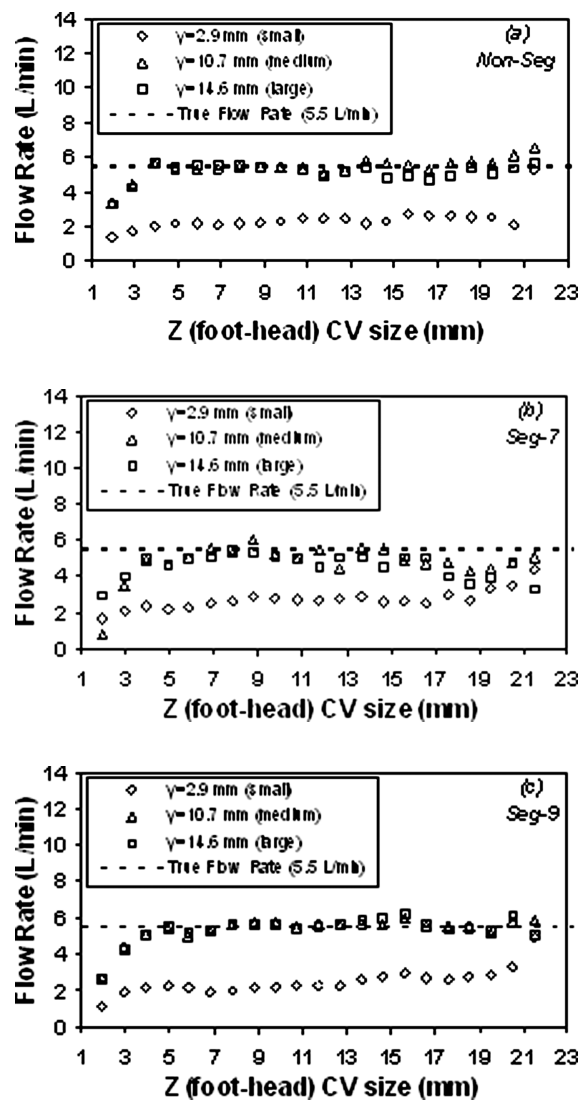

FIGURE 5. Measured flow rates for a variety of CV sizes in the foot-head ( $z$ ) and anterior-posterior $(y)$ direction through a $5 \mathrm{~mm}$ ID circular orifice for a true regurgitant flow rate of 5.5 $\mathrm{I} / \mathrm{min}$. The $\mathrm{x}$-size was $15 \mathrm{~mm}$ (three slices); 2 (a) nonseg; (b) seg-7; and (c) seg-9.

posterior) CV sizes. Figure 5(a) corresponds to the nonsegmented MR-PVM acquisition, whereas Figs. 5(b) and 5(c) correspond to the segmented acquisitions with seven and nine lines per segment, respectively. The $x$-size of the $\mathrm{CV}$ was $15 \mathrm{~mm}$ (three slices). The true regurgitant flow rate for this case was $5.5 \mathrm{l} / \mathrm{min}$ and there was no aortic outflow. As seen, in all acquisitions, the measured flow rate underestimated the true value when the $y$-size of the CV was small [ $2.9 \mathrm{~mm}$ in the particular case of Fig. $5(\mathrm{a}-\mathrm{c})]$ and for small $z$-size $(<3.9 \mathrm{~mm}$ for this case). This error was as high as $60 \%$ considering all orifices and flow cases studied. As the $z$-size increased ( $\geq 3.9 \mathrm{~mm}$ in this case), the flow rate data formed a plateau. The plateau underestimated (errors as high as 30\%) the true flow rate for a CV of small $y$-size ( $2.9 \mathrm{~mm}$ in this case), but was very close to the true flow rate line for greater $y$-sizes (10.7 or $14.6 \mathrm{~mm}$ in Fig. 5) with an average error $<2 \%$. As the CV size increased, some disturbance in the plateau was observed. This can be seen in Fig. 5 for $z>15 \mathrm{~mm}$ and also in Fig. 6, which shows the change in the measured flow rate with the $y$-size of the $\mathrm{CV}$ for three different $z$-sizes. As seen, the accuracy of the flow 


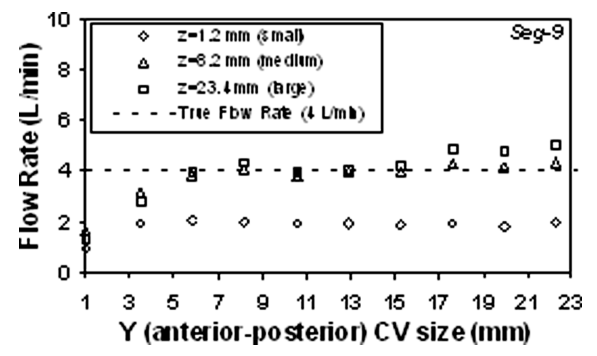

FIGURE 6. Measured flow rates using the seg-9 acquisition for a variety of $\mathrm{CV}$ sizes in the anterior-posterior $(y)$ and foothead (z) direction through a $5 \mathrm{~mm}$ ID circular orifice for a true regurgitant flow rate of $4 \mathrm{l} / \mathrm{min}$. The $x$-size was $15 \mathrm{~mm}$ (three slices).

rate measurements decreased when the $\mathrm{CV}$ size became too large (errors as high as $40 \%$ ).

No negative effects from the presence of aortic outflow can be seen in Fig. 7, which shows the calculated regurgitant flow rate through the $3 \mathrm{~mm}$ ID circular orifice for a true regurgitant flow rate of $1.0 \mathrm{l} / \mathrm{min}$ with aortic outflow rates of 0,10 and $25 \mathrm{l} / \mathrm{min}$. In this case, there was no significant underestimation of the flow rate for very small $z$ values, because of the small size of the orifice, which restricted the region of flow underestimation to a distance $<3 \mathrm{~mm}$. A well-shaped plateau is seen for $z$ between 7 and $13 \mathrm{~mm}$ with values very close to the true flow rate line.

Distinct plateaus were observed in the "flow rate" vs. "distance" plots, regardless of the presence or absence of aortic outflow, for all orifices and flow cases studied. The range of $z$-size and $y$-size for which the flow rate data showed a plateau varied depending on the orifice used and on the magnitude of the regurgitant flow rate, as seen in Table 3. Three slices in the $x$ direction $(15 \mathrm{~mm})$ provided more accurate results in the circular orifices, but five slices $(25 \mathrm{~mm})$ were necessary for accurate results in the slit-like orifice. Tables 4 and 5 show the measured flow rates (as averaged values from the plateaus corresponding to the size ranges found in Table 3 ) and the percent errors with respect to the true flow rates for all orifices and flow rates used, without and with aortic outflow, respectively. As seen, the

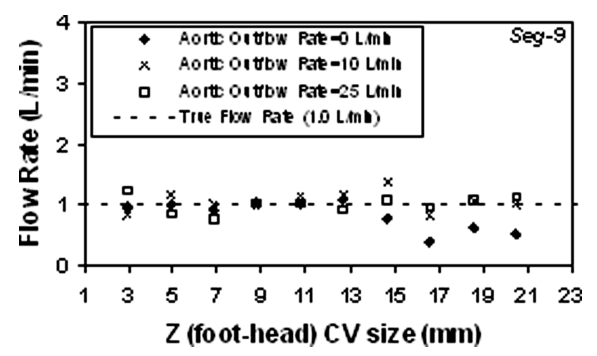

FIGURE 7. Calculated regurgitant flow rate through the $3 \mathrm{~mm}$ ID circular regurgitant orifice under the presence of 0,10 , and $25 \mathrm{l} / \mathrm{min}$ aortic outflow, using the seg-9 MR-PVM sequence.
TABLE 3. Summary of the ranges of CV size for accurate regurgitant flow rate measurements.

\begin{tabular}{lccc}
\hline & \multicolumn{3}{c}{ CV dimension (mm) } \\
\cline { 2 - 4 } Orifice geometry & $\begin{array}{c}x \\
\text { (left-right) }\end{array}$ & $\begin{array}{c}y \\
\text { (anterior-posterior) }\end{array}$ & $\begin{array}{c}z \\
\text { (foot-head) }\end{array}$ \\
\hline Circular, ID of $5 \mathrm{~mm}$ & 15 & $11-17$ & $6-11$ \\
Circular, ID of $3 \mathrm{~mm}$ & 15 & $7-17$ & $5-11$ \\
Slit-like, $4 \times 10 \mathrm{~mm}^{2}$ & 25 & $9-19$ & $6-13$ \\
\hline
\end{tabular}

average errors were small for all sequences used with and without aortic outflow.

The pulsatile flow results showed similar features to the steady flow results. Figure 8 shows the measured flow waveforms through the $3 \mathrm{~mm}$ ID circular orifice for a true regurgitant flow volume of $10 \mathrm{ml}$ per cycle (a), and through the $4 \times 10 \mathrm{~mm}$ slit-like orifice for a true regurgitant flow volume of $40 \mathrm{ml}$ per cycle (b), and $60 \mathrm{ml}$ per cycle (c), using the nonseg, seg-7, and seg-9 sequences. The flow rates were calculated as the average values in the plateaus for a $y$-size between 7 and $17 \mathrm{~mm}$, an $x$-size of $15 \mathrm{~mm}$ (three slices), and a $z$-size between 5 and $11 \mathrm{~mm}$ in the circular orifice; and for a $y$-size between 9 and $19 \mathrm{~mm}$, an $x$-size of $25 \mathrm{~mm}$ (five slices), and a $z$-size between 6 and $13 \mathrm{~mm}$ in the slitlike orifice. As seen, there is general agreement between the data points from all three sequences used. Table 6 summarizes the comparison between the calculated regurgitant flow volumes (integration during systole) for all orifices and all sequences used. As seen, the measured flow volumes with the segmented (seg-7 and seg-9) techniques are in agreement with the nonseg ones, as well as with the true flow volumes regardless of the orifice shape and size. All sequences provided results with similar low errors (average error $<5 \%$ ).

Statistical analysis with $t$-tests failed to show any significant differences between the measured flow rates with any of the three sequences and the true flow values, with or without aortic outflow ( $p$-values $>0.05$ ). Similarly, $t$-tests failed to show any significant differences between the segmented sequences and the nonsegmented sequence (again all $p$-values $>0.05$ ). Analysis of variance failed to show the presence of significant differences in the results between the three sequences and the three orifices ( $p=0.771$ for the sequence factor, $p=0.229$ for the orifice factor, and $p=0.960$ for the combined "sequence* orifice" factor, without aortic outflow). Analysis of variance also failed to show any significant difference between the flow rate results with and without aortic outflow ( $p=0.622)$. Similarly, in pulsatile flow, sign tests failed to show any significant differences between the calculated regurgitant flow volumes with any of the three sequences and the true flow values ( $p$-values $>0.05$ ). A Kruskal-Wallis test failed to show any significant effect from the orifice geometry ( $p$-value $=$ $0.193)$. 
TABLE 4. Summary of MR-PVM measured regurgitant flow rate results without the presence of aortic outflow.

\begin{tabular}{|c|c|c|c|c|c|c|c|}
\hline \multirow[b]{3}{*}{ Orifice geometry } & \multirow[b]{3}{*}{ True flow rate $(\mathrm{l} / \mathrm{min})$} & \multicolumn{6}{|c|}{ Measured flow rate ( $\mathrm{l} / \mathrm{min})$ and errors (\%) } \\
\hline & & \multicolumn{2}{|c|}{ Nonseg ${ }^{\mathrm{a}}$} & \multicolumn{2}{|c|}{ Seg- $7^{b}$} & \multicolumn{2}{|c|}{ Seg- $9^{c}$} \\
\hline & & Flow & Error & Flow & Error & Flow & Error \\
\hline \multirow{3}{*}{ Circular ID of $5 \mathrm{~mm}$} & 2.5 & 2.4 & -3.8 & 2.4 & -3.9 & 2.5 & 1.5 \\
\hline & 4.0 & 4.2 & 4.1 & 4.3 & 6.3 & 4.1 & 3.0 \\
\hline & 5.5 & 5.4 & -1.6 & 5.3 & -4.1 & 5.6 & 1.7 \\
\hline \multirow[t]{3}{*}{ Circular ID of $3 \mathrm{~mm}$} & 1.0 & 1.1 & 8.1 & 1.2 & 21.6 & 1.0 & -3.0 \\
\hline & 1.5 & 1.4 & -5.6 & 1.4 & -5.0 & 1.5 & -0.5 \\
\hline & 2.0 & 2.3 & 15.0 & 1.8 & -12.5 & 2.1 & 6.5 \\
\hline \multirow[t]{3}{*}{ Slit-like $4 \times 10 \mathrm{~mm}^{2}$} & 4.5 & 4.3 & -4.0 & 4.2 & -6.1 & 4.3 & -4.4 \\
\hline & 7.0 & 7.2 & 2.7 & 6.7 & -4.4 & 6.7 & -4.0 \\
\hline & 11.0 & 10.6 & -3.4 & 10.7 & -3.0 & 10.8 & -1.6 \\
\hline Mean Error (\%) & & \multicolumn{2}{|c|}{1.3} & \multicolumn{2}{|c|}{-1.2} & \multicolumn{2}{|c|}{-0.1} \\
\hline
\end{tabular}

aNonsegmented.

bSegmented with seven lines per segment.

'Segmented with nine lines per segment.

TABLE 5. Summary of MR-PVM measured regurgitant flow rate results with the presence of aortic outflow.

\begin{tabular}{|c|c|c|c|c|c|c|c|c|}
\hline \multirow[b]{3}{*}{ Orifice geometry } & \multirow[b]{3}{*}{ True flow rate $(\mathrm{l} / \mathrm{min})$} & \multirow[b]{3}{*}{ Aortic outflow (I/min) } & \multicolumn{6}{|c|}{ Measured flow rate $(1 / \mathrm{min})$ and errors (\%) } \\
\hline & & & \multicolumn{2}{|c|}{ Nonseg ${ }^{\mathrm{a}}$} & \multicolumn{2}{|c|}{ Seg- $\not 0$} & \multicolumn{2}{|c|}{ Seg-9c } \\
\hline & & & Flow & Error & Flow & Error & Flow & Error \\
\hline \multirow[t]{4}{*}{ Circular ID of $5 \mathrm{~mm}$} & 2.5 & 10 & 2.8 & 11.2 & 2.5 & 0.7 & 2.2 & -12.6 \\
\hline & & 25 & 2.4 & -6.1 & 2.3 & -7.4 & 2.2 & -11.7 \\
\hline & 5.5 & 10 & 5.5 & -0.1 & 5.7 & 3.2 & 5.6 & 1.6 \\
\hline & & 20 & 5.6 & 1.1 & 5.3 & -3.1 & 5.1 & -7.4 \\
\hline \multirow[t]{3}{*}{ Circular ID of $3 \mathrm{~mm}$} & 1.0 & 10 & 1.0 & -5.3 & 1.1 & 12.2 & 1.1 & 13.3 \\
\hline & & 25 & 1.0 & -4.9 & 1.1 & 8.4 & 1.0 & -3.6 \\
\hline & 2.0 & 5 & 2.1 & 2.7 & 2.2 & 10.4 & 2.1 & 3.7 \\
\hline \multirow[t]{3}{*}{ Slit-like $4 \times 10 \mathrm{~mm}^{2}$} & 4.5 & 10 & 4.5 & -1.1 & 4.6 & 1.1 & 3.7 & -16.9 \\
\hline & & 25 & 4.7 & 3.6 & 4.0 & -11.6 & 4.3 & -5.2 \\
\hline & 11.0 & 10 & 10.8 & -2.1 & 10.6 & -3.5 & 9.5 & -13.4 \\
\hline \multicolumn{3}{|c|}{ Mean Error with true flow rate (\%) } & \multicolumn{2}{|c|}{-0.1} & \multicolumn{2}{|c|}{1.0} & \multicolumn{2}{|c|}{-5.2} \\
\hline
\end{tabular}

aNonsegmented.

bSegmented with seven lines per segment.

cSegmented with nine lines per segment.

TABLE 6. Summary of MR-PVM measured regurgitant flow volume results under pulsatile flow.

\begin{tabular}{|c|c|c|c|c|c|c|c|}
\hline \multirow[b]{3}{*}{ Orifice geometry } & \multirow[b]{3}{*}{ True flow volume (ml per cycle) } & \multicolumn{6}{|c|}{ Calculated flow volume (ml per cycle) and error (\%) } \\
\hline & & \multicolumn{2}{|c|}{ Nonseg ${ }^{\mathrm{a}}$} & \multicolumn{2}{|c|}{ Seg- $7^{b}$} & \multicolumn{2}{|c|}{ Seg-gc } \\
\hline & & Flow & Error & Flow & Error & Flow & Error \\
\hline Circular ID of $5 \mathrm{~mm}$ & 30 & 29.4 & -2.0 & 30.5 & 1.5 & 28.3 & -5.7 \\
\hline Circular ID of $3 \mathrm{~mm}$ & 10 & 12.0 & 19.6 & 11.5 & 14.7 & 9.9 & -0.9 \\
\hline \multirow[t]{2}{*}{ Slit-like $4 \times 10 \mathrm{~mm}^{2}$} & 40 & 40.8 & 2.1 & 41.6 & 3.9 & 41.9 & 4.7 \\
\hline & 60 & 57.4 & -4.3 & 58.2 & -3.1 & 56.5 & -5.9 \\
\hline \multicolumn{2}{|c|}{ Mean error with true flow volume (\%) } & \multicolumn{2}{|c|}{3.9} & \multicolumn{2}{|c|}{4.3} & \multicolumn{2}{|c|}{-2.0} \\
\hline
\end{tabular}

\footnotetext{
aNonsegmented.

${ }^{b}$ Segmented with seven lines per segment.

cSegmented with nine lines per segment.
} 

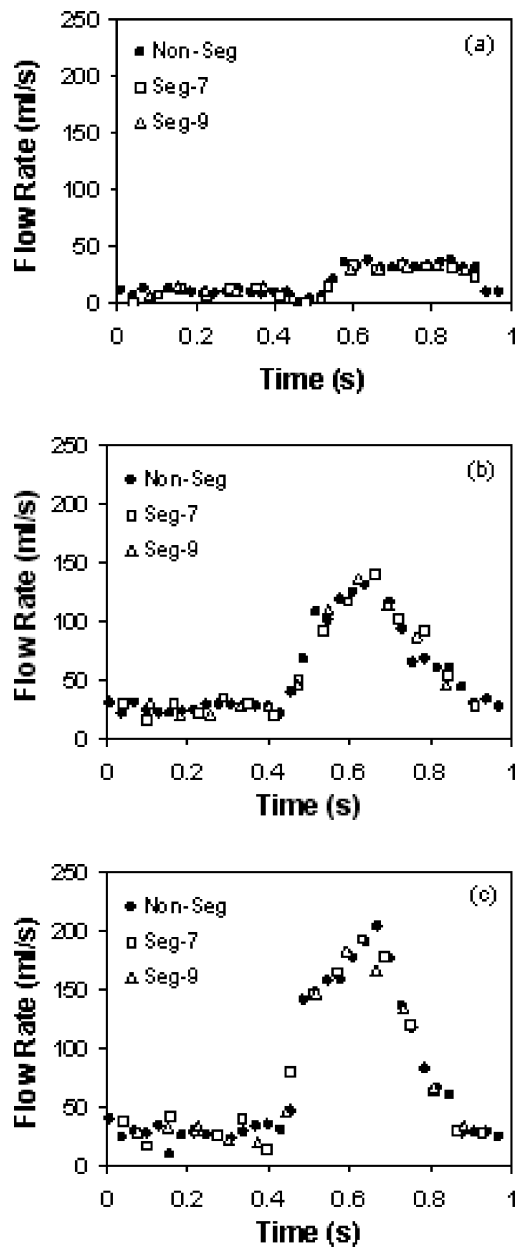

FIGURE 8. Flow profiles through a $3 \mathrm{~mm}$ ID circular orifice for a true flow volume of $10 \mathrm{ml}$ per cycle (a), and through a $4 \times 10$ slit-like for a true flow volume of $40 \mathrm{ml}$ per cycle (b) and $60 \mathrm{ml}$ per cycle (c) orifice using the nonseg, seg-7, and seg-9.

\section{DISCUSSION}

Quantitative information of the severity of mitral regurgitation is important to better monitor the progression of the disease. However, due to the complex flow environment close to the regurgitant mitral orifice in the LV cavity, there has been a lack of reliable noninvasive clinical techniques to provide the regurgitant flow.

The CV method was developed to overcome the limitation of MR-PVM of not being able to accurately quantify flows immediately upstream and downstream of narrow orifices (such as the case of a regurgitant heart valve). This limitation is related to the signal loss both upstream and downstream to the orifice. The signal loss causes velocity errors in those regions. In upstream, the signal loss is caused by intense flow acceleration as blood converges to pass through the orifice. In downstream, the signal loss is caused by the turbulent jet that emerges. It becomes difficult to accurately measure the velocity exactly at the orifice (tip of the valve leaflets), because an imaging slice passing through the orifice would include some of the problematic upstream and some of the downstream regions. The lack of reliable regurgitant velocity data exactly at the tip of the valve leaflets during systole complicates the quantification of quantities such as the regurgitant pressure gradient through the mitral regurgitant valve (via the Bernoulli equation) using MR-PVM.

The CV method overcomes the limitation as mentioned earlier, because the quantification of the regurgitant flow takes place outside the region of signal loss using the principle of mass conservation. The only essential assumption is that the flow must be incompressible, which is valid in the case of blood in the left ventricle. Therefore, the accuracy of the CV method depends on the accuracy of the velocity measurements. A multislice CV method implemented with the nonsegmented MR-PVM has shown great potential in vitro to quantify the mitral regurgitant volume. ${ }^{5}$ Despite its accuracy, the long duration of nonsegmented MR-PVM (approximately $1 \mathrm{~h}$ for the acquisition of all three velocity components in five slices) prevents the clinical implementation of the technique within a routine cardiac MR examination. Faster techniques, such as the $k$-space segmented MR-PVM, show great potential to overcome this problem. Using a sequence with nine lines per segment, it takes $<10$ min (including preparation time between each acquisition) for a five-slice, three-directional velocity acquisition. Based on our previous accurate results in straight tubes ${ }^{30,31}$ and in the human aorta, ${ }^{6}$ we performed this study to determine in vitro whether segmented $k$-space MR-PVM has potential to quantify the regurgitant flow using the $\mathrm{CV}$ method.

The acquired images (both magnitude and phase) were of sufficient quality for a visual identification of the orifice and the other geometrical features of the model. The CV size was varied to investigate whether it had any effect on the calculated flow rate. The results showed that a small CV $(z<5 \mathrm{~mm}$ and $y<9 \mathrm{~mm}$, for most of the orifice and flow cases studied in this study) causes underestimation of the regurgitant flow, because the faces of the CV include voxels with signal loss (due to the flow acceleration). Therefore, the calculated flow rate underestimates the true regurgitant flow rate. In addition, too close to the orifice, there is aliasing of the velocity as a result of the large local velocity and selection of a velocity-encoding value smaller that the orifice velocity, in order to increase the sensitivity of the scanner in measuring the local velocity at medium distance from the orifice.

For a larger CV (medium size) in the $z$ and $y$ directions, the flow rate data showed plateaus. Calculation of an average flow rate in these plateaus provided accurate flow quantifications of the true regurgitant flow rates (errors $\leq 5 \%$ ). Statistical analysis also showed that there is no significant difference between the true flow rate and the MR-PVM measured flow rate with all three different sequences (all $p$-values $>0.05)$. Although the term "medium-sized" is 
qualitative, in practice, it corresponds to a CV large enough for its boundaries to be outside the region of signal loss very close to the orifice due to rapid acceleration and velocity aliasing.

When the CV size was too large $(z>13 \mathrm{~mm}$ and $y>19 \mathrm{~mm}$, for most of the orifice and flow cases studied in this study), the results showed deviations from the true flow values (errors as high as $40 \%$ ) probably due to the lower velocity sensitivity far away from the orifice (the local velocity away from the orifice was much smaller than the velocity-encoding values).

Both segmented MR-PVM sequences showed similar levels of accuracy (compared with the true flow data) and close agreement to the nonsegmented sequence, reducing significantly the data acquisition time from approximately $1 \mathrm{~h}$ to a few minutes. This result is very important because it shows that in addition to its accuracy, MR-PVM can be applied rapidly and become part of a routine MR examination. The shape and size of the orifice did not affect the accuracy of the measurements, although it affected the extent of the signal loss region close to the orifice, which must be avoided by the boundaries of the CV and, thus the optimal range of $\mathrm{CV}$ sizes (where the plateaus were formed). For a large orifice, a corresponding large $\mathrm{CV}$ is needed in order to cover the orifice entirely and be of sufficient distance from the region of signal loss. For the $4 \times 10 \mathrm{~mm}$ slit-like orifice, placed in a way such that its large size be aligned with the $x$ (left-right) direction of the $\mathrm{CV}$, a size of $25 \mathrm{~mm}$ (five slices) was necessary in the $x$ direction compared with the $15 \mathrm{~mm}$ (three slices) for the circular orifices. The reason for such a behavior was that the acceleration region extended further into the ventricular region, especially in the $x$ direction, and caused underestimation of the flow rate further from the orifice.

The effect of the aortic outflow on the accuracy of the calculated flow rate through a regurgitant orifice was evaluated by varying the magnitude of the aortic outflow under a variety of regurgitant flow conditions and orifice shapes/sizes. As shown in Fig. 7, the data formed plateaus similarly to the case without aortic outflow. The effect of aortic outflow is shown in Fig. 9. Some of the fluid that leaves the ventricle crosses the $\mathrm{CV}$ boundaries. Theoretically, based on the mass conservation principle, any inflow to the $\mathrm{CV}$ that does not pass through the regurgitant orifice will flow out of the CV (cancel-out effect); therefore, the presence of aortic outflow should not affect the implementation of the $\mathrm{CV}$ method. The results of this study support this principle (all $p$-values $>0.05$ ).

From the pulsatile flow studies (Fig. 8), it can be seen clearly that the flow profiles measured with all three sequences agreed very closely during the entire cycle. The calculated values for the regurgitant flow volumes for all sequences (determined by integrating the flow curves during "systole") agreed very closely with the true flow volumes (Table 6). Analysis of variance also showed that the type of

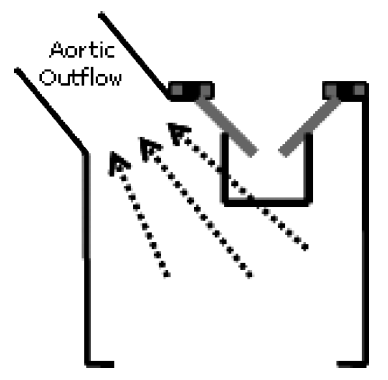

FIGURE 9. Interaction of aortic outflow with the CV. Some of the ventricular blood enters and exits the boundaries of the $\mathrm{CV}$ before it flows out through the aorta.

sequence (nonseg, seg-7, or seg-9) and the type of orifice geometry $\left(3 \mathrm{~mm}\right.$ circular, $5 \mathrm{~mm}$ circular, or $4 \times 10 \mathrm{~mm}^{2}$ slit-like) have no statistically significant effect on the quantification of the pulsatile flow through the orifice using the CV method ( $p$-values $>0.05$ ). Similarly to the steady flow studies, the measurements of the regurgitant flow rate at each time-phase of the cycle were inaccurate (errors up to $50 \%$ ) when the $\mathrm{CV}$ was too small and its boundaries were inside the signal loss region. When the $\mathrm{CV}$ was large enough for its boundaries to be outside this region of signal loss, the measurements were very accurate (Table 6). Because the pulsatile flow studies were performed under the presence of aortic outflow, the accuracy of the regurgitant volume results serves as another indication for the absence of any significant effect from the aortic outflow on the accuracy of the $\mathrm{CV}$ calculations.

This study focused on the effects of fast image acquisition and on the effects of the flow environment on the accuracy of the $\mathrm{CV}$ method. Future studies will focus on additional factors, such as the presence and motion of the chordae tendineae, in order to determine their effects on the reliability of the $\mathrm{CV}$ method, as well as valve and heart motion. ${ }^{29}$ These studies should be followed by clinical studies in order to focus on additional clinical factors before this method becomes clinically applicable. It is of importance, however, that the present study showed that the CV method can be applied rapidly, thus eliminating one of its previously reported limitations (long scan duration). It should be noted that clinically, in cases of mitral valve regurgitation, the flow acceleration region upstream to the regurgitant orifice can be seen as a (hemispherical-like) signal loss region. According to the results of the present study, this signal loss region should guide the placement of the $\mathrm{CV}$ clinically in order to avoid errors in the velocity measurement. By selecting a CV large enough so that its boundaries exclude this signal loss region, the regurgitant flow should be accurately quantified.

In conclusion, $k$-space segmented MR-PVM can rapidly and accurately quantify the flow through orifices simulating regurgitant mitral valves in vitro, and therefore, shows great clinical potential. The size of the $\mathrm{CV}$ is of importance 
because the boundaries of the CV should be outside the region of velocity aliasing and flow acceleration very close to the orifice, in order to avoid errors in the velocity acquisitions.

\section{ACKNOWLEDGMENTS}

We would like to thank David Epperly for his skillful work in constructing the mitral regurgitant models. Support by Cleveland State University is greatly appreciated.

\section{REFERENCES}

${ }^{1}$ Bogren, H. G., and M. H. Buonocore. Blood flow measurements in the aorta and major arteries with MR velocity mapping. $J$. Magn. Reson. Imag. 4:119-130, 1994.

${ }^{2}$ Bryant, D. J., J. A. Payne, D. N. Firmin, and D. B. Longmore. Measurement of flow with NMR imaging using a gradient pulse and phase difference technique. J. Comp. Assist. Tomogr. 8:588593, 1984

${ }^{3}$ Cape, E. G., Y. H. Kim, R. S. Heinrich, R. Y. Grimes, E. Muralidharan, J. D. Broder, E. Schwammenthal, A. P. Yoganathan, and R. A. Levine. Cardiac motion can alter proximal isovelocity surface-area calculations of regurgitant flow.J. Am. Col. Cardiol. 22:1730-1737, 1993.

${ }^{4}$ Chatzimavroudis, G. P., P. G. Walker, J. N. Oshinski, R. H. Franch, R. I. Pettigrew, and A. P. Yoganathan. Slice location dependence of aortic regurgitation measurements with MR phase velocity mapping. Magn. Reson. Med. 37:545-551, 1997.

${ }^{5}$ Chatzimavroudis, G. P., J. N. Oshinski, R. I. Pettigrew, P. G. Walker, R. H. Franch, and A. P. Yoganathan. Quantification of mitral regurgitation with magnetic resonance phase velocity mapping using a control volume method. J. Magn. Reson. Imag. 8:577-582, 1998.

${ }^{6}$ Chatzimavroudis, G. P., H. Zhang, S. S. Halliburton, J. R. Moore, O. P. Simonetti, P. R. Schvartzman, A. E. Stillman, and R. D. White. Clinical blood flow quantification with segmented $k$ space magnetic resonance phase velocity mapping. J. Magn. Reson. Imag. 17:65-71, 2003.

${ }^{7}$ Chatzimavroudis, G. P., J. N. Oshinski, R. H. Franch, P. G. Walker, A. P. Yoganathan, and R. I. Pettigrew. Evaluation of the precision of magnetic resonance phase velocity mapping for blood flow measurements. J. Cardiovasc. Magn. Reson. 3:1119, 2001.

${ }^{8}$ Croft, C. H., K. Lipscomb, K. Mathis, B. G. Firth, P. Nicod, G. Tilton, M. D. Winniford, and L. D. Hillis. Limitations of qualitative angiographic grading in aortic or mitral regurgitation. Am. J. Cardiol. 53:1593-1598, 1984.

${ }^{9}$ Dulce, M. C., G. H. Mostbeck, M. O'Sullivan, M. Cheitlin, G. R. Caputo, and C. B. Higgins. Severity of aortic regurgitation: Interstudy reproducibility of measurements with velocity-encoded cine MR imaging. Radiology 185:235-240, 1992.

${ }^{10}$ Frayne, R., D. A. Steinman, C. R. Ethier, and B. K. Rutt. Accuracy of MR phase contrast velocity measurements for unsteady flow. J. Magn. Reson. Imag. 5:428-431, 1995.

${ }^{11}$ Hopmeyer, J., A. A. Fontaine, S. Yang, R. A. Levine, and A. P. Yoganathan. The effect of aortic outflow on the quantification of mitral regurgitation by the flow convergence method. J. Am. Soc. Echo. 9:44-57, 1996.

${ }^{12}$ Kilner, P. J., G. Z. Yang, R. H. Mohiaddin, D. N. Firmin, and D. B. Longmore. Helical and retrograde secondary flow patterns in the aortic arch studied by three-directional magnetic resonance velocity mapping. Circulation 88:2235-2247, 1993.
${ }^{13}$ Klipstein, R. H., D. N. Firmin, S. R. Underwood, R. S. Rees, and D. B. Longmore. Blood flow patterns in the human aorta studied by magnetic resonance. Br. Heart J. 58:316-323, 1987.

${ }^{14}$ Kondo, C., G. R. Caputo, R. Semelka, E. Foster, A. Shimakawa, and C. B. Higgins. Right and left ventricular stroke volume measurements with velocity-encoded cine MR imaging: In-vitro and in-vivo validation. Am. J. Roentgenol. 157:9-16, 1991.

${ }^{15}$ Kraft, K. A., D. Y. Fei, and P. P. Fatouros. Quantitative phasevelocity MR imaging of in-plane laminar flow: Effect of fluid velocity, vessel diameter, and slice thickness. Med. Phys. 19:7985, 1992.

${ }^{16}$ McKinnon, G. C., J. F. Debatin, D. R. Wetter, and G. K. Von Schulthess. Interleaved echo planar flow quantitation. Magn. Reson. Med. 32:263-267, 1994.

${ }^{17}$ Meier, D., S. Maier, and P. Bosiger. Quantitative flow measurements on phantoms and on blood vessels with MR. Magn. Reson. Med. 8:25-34, 1988.

${ }^{18}$ Mohiaddin, R. H., S. L. Wann, R. Underwood, D. N. Firmin, S. Rees, and D. B. Longmore. Vena caval flow: Assessment with cine MR velocity mapping. Radiology 177:537-541, 1990.

${ }^{19}$ Moran, P. R. A flow velocity zeugmatographic interlace for NMR imaging in humans. Magn. Reson. Imag. 1:197-203, 1982.

${ }^{20}$ Pelc, L. R., N. J. Pelc, S. C. Rayhill, L. J. Castro, G. H. Glover, R. J. Herfkens, D. C. Miller, and R. B. Jeffrey. Arterial and venous blood flow: Noninvasive quantification with MR imaging. Radiology 185:809-812, 1992.

${ }^{21}$ Perry, G. J., F. Helmcke, N. C. Nanda, C. Byard, and B. Soto. Evaluation of aortic insufficiency by Doppler color flow mapping. J. Am. Coll. Cardiol. 9:952-959, 1987.

${ }^{22}$ Recusani, F., G. S. Bargiggia, A. P. Yoganathan, A. Raisaro, L. M. Valdes-Cruz, H. W. Sung, C. Bertucci, M. Gallati, V. A. Moises, I. A. Simpson, L. Tronconi, and D. J. Sahn. A new method for quantification of regurgitant flow rate using color Doppler flow imaging of the flow convergence region proximal to a discrete orifice. An in vitro Study Circulation 83:594-604, 1991.

${ }^{23}$ Sechtem, U., P. W. Pflugfelder, M. M. Cassidy, R. D. White, M. D. Cheitlin, N. B. Schiller, and C. B. Higgins. Mitral or aortic regurgitation: Quantification of regurgitant volumes with cine MR imaging. Radiology 167:425-430, 1988.

${ }^{24}$ Suzuki, J., G. R. Caputo, C. Kondo, and C. B. Higgins. Cine MR imaging of valvular heart disease: Display and imaging parameters affect the size of the signal void caused by valvular regurgitation. Am. J. Roentgenol. 155:723-727, 1990.

${ }^{25}$ Thomsen, C., M. Cortsen, L. Sondergaard, O. Henriksen, and F. Stahlberg. A segmented $k$-space velocity mapping protocol for quantification of renal artery blood flow during breath-holding. J. Magn. Reson. Imag. 5:393-401, 1995.

${ }^{26}$ Underwood, S. R., D. N. Firmin, R. H. Klipstein, R. S. Rees, and D. B. Longmore. Magnetic resonance velocity mapping: Clinical application of a new technique. Br. Heart J. 57:404-412, 1987.

${ }^{27}$ Veyrat, C., A. Ameur, C. Gourtchiglouian, A. Lessana, G. Abitbol, and D. Kalmanson. Calculation of pulsed Doppler left ventricular outflow tract regurgitant index for grading the severity of aortic regurgitation. Am. Heart J. 108:507-515, 1984.

${ }^{28}$ Walker, P. G., S. Oyre, E. M. Pedersen, K. Houlind, F. S. Guenet, and A. P. Yoganathan. A new control volume method for calculating valvular regurgitation. Circulation 92:579-586, 1995.

${ }^{29}$ Walker, P. G., K. Houlind, C. Djurhuus, W. Y. Kim, and E. M. Pedersen. Motion correction for the quantification of mitral regurgitation using the control volume method. Magn. Reson. Med. 43:726-733, 2000. 
${ }^{30}$ Zhang, H., S. S. Halliburton, J. R. Moore, O. P. Simonetti, P. R. Schvartzman, R. D. White, and G. P. Chatzimavroudis. Ultra-fast flow quantification with segmented $k$-space magnetic resonance phase velocity mapping. Ann. Biomed. Eng. 30:120-128, 2002.
${ }^{31}$ Zhang, H., S. S. Halliburton, J. R. Moore, O. P. Simonetti, R. D. White, and G. P. Chatzimavroudis. Evaluation of the accuracy of in-plane velocity measurements with segmented $k$-space magnetic resonance phase velocity mapping. BMES Annual Meeting, Houston, TX, 2002. 